\title{
ON NONLINEAR SCALAR VOLTERRA INTEGRAL EQUATIONS. I
}

\author{
BY
}

HANS ENGLER

ABSTRACT. The scalar nonlinear Volterra integral equation

$$
u(t)+\int_{0}^{t} g(t, s, u(s)) d s=f(t) \quad(0 \leqslant t)
$$

is studied. Conditions are given under which the difference of two solutions can be estimated by the variation of the difference of the corresponding right-hand sides. Criteria for the existence of $\lim u(t)($ as $t \rightarrow \infty)$ are given, and existence and uniqueness questions are also studied.

0. Consider the scalar ordinary differential equation

$$
u^{\prime}(t)+g(u(t))=f^{\prime}(t) \quad(0 \leqslant t \leqslant T)
$$

together with some initial condition $u(0)=u_{0}$. The following facts are well known:

(i) If $g$ is monotone nondecreasing and $u_{1}(\cdot), u_{2}(\cdot)$ are two solutions of $(0.1)$ with right-hand sides $f_{1}^{\prime}, f_{2}^{\prime}$ in $L^{1}(0, T ; \mathbf{R})$, then for any $t \in[0, T]$

$$
\left|u_{1}(t)-u_{2}(t)\right| \leqslant\left|u_{1}(0)-u_{2}(0)\right|+\int_{0}^{t}\left|f_{1}^{\prime}(s)-f_{2}^{\prime}(s)\right| d s .
$$

(ii) If $g=g_{0}+g_{1}, g_{0}$ being monotone nondecreasing and $g_{1}$ locally Lipschitz-continuous, then $(0.1)$ together with an initial condition has at most one solution for any $f^{\prime}$ in $L^{1}(0, T ; \mathbf{R})$.

(iii) If $g(u)=g_{0}(u)-L \cdot u, g_{0}$ monotone nondecreasing and $L$ some nonnegative number, then (0.1) has in fact a unique solution $u$ for any $f^{\prime}$ that is the distributional derivative of an essentially bounded function, and $u-f$ is absolutely continuous.

(iv) Assume the situation described in (i); then

$$
\int_{0}^{T}\left|u_{1}^{\prime}(s)-u_{2}^{\prime}(s)\right| d s \leqslant\left|u_{1}(0)-u_{2}(0)\right|+2 \cdot \int_{0}^{T}\left|f_{1}^{\prime}(s)-f_{2}^{\prime}(s)\right| d s .
$$

(v) Assume the situation described in (i). The conditions

$$
\begin{gathered}
f_{1}^{\prime}(t) \leqslant f_{2}^{\prime}(t) \quad \text { for a.e. } t, \\
u_{1}(0) \leqslant u_{2}(0)
\end{gathered}
$$

Received by the editors December 6, 1983 and, in revised form, December 4, 1984.

1980 Mathematics Subject Classification. Primary 45D05.

Key words and phrases. Nonlinear scalar Volterra equation, uniqueness, existence, continuous dependence on data, a priori estimates, asymptotic behavior, log-convex kernels. 
imply

$$
u_{1}(t) \leqslant u_{2}(t) \text { for all } t \in[0, T] .
$$

A simple proof leading to $(0.2)$ and $(0.3)$ consists in subtracting the equations for $u_{1}$ and for $u_{2}$ from each other, multiplying the resulting identity for each $t$ with $\operatorname{sign}\left(u_{1}(t)-u_{2}(t)\right)$, and integrating from 0 to any fixed $t$. This gives $(0.2)$ and an estimate for

$$
\int_{0}^{t}\left|g\left(u_{1}(s)\right)-g\left(u_{2}(s)\right)\right| d s,
$$

from which (0.3) follows. The uniqueness property (ii) follows in a standard manner by using Gronwall's lemma and (0.2), and (iii) can be shown by considering the equation for $v=u-f$,

$$
v^{\prime}(t)+g(v(t)+f(t))=0,
$$

and using standard local existence arguments [10] together with an estimate of the type (0.2) for $|v(t)|=|v(t)-0|$. The comparison statement (v) is a consequence of the fact "unique solutions do not cross". In this and in a following note, we want to find corresponding conditions such that conclusions like (i)-(v) are true for solutions of the scalar Volterra integral equation

$$
u(t)+\int_{0}^{t} g(t, s, u(s)) d s=f(t) .
$$

The more special cases

$$
\begin{gathered}
g(t, s, u)=\sum_{i=1}^{N} a_{i}(t, s) k_{i}(s, u), \\
g(t, s, u)=\bar{g}(t-s, u), \\
g(t, s, u)=\sum_{i=1}^{N} a_{i}(t-s) \bar{k}_{i}(u)
\end{gathered}
$$

will also be discussed. In the following $\$ \S 1-3$, we discuss conditions leading to analogues of (ii), (i), (iii), respectively (in this order). In a following note, some analogues of (iv) and (v) will be given. Frequent use will be made of various transformations of $(0.6)$, such as:

multiplication of (0.6) with a scalar function $\alpha(t)$,

convolution of $(0.6)$ with a scalar function $b(t)$ and addition of the resulting equation and (0.6),

introduction of the new unknown function $v(t)=u(t)-f(t)$.

It is the stability of the equation of type (0.6) under these transformations that turns out to be advantageous for a discussion of this class of equations. Nevertheless, it seems that many of the results in this note are new even for the class of the equations of the form

$$
u(t)+\int_{0}^{t} a(t-s) \bar{k}(u(s)) d s=f(t)
$$


which has been discussed extensively in the literature. We shall only discuss the case where $g(t, t, \cdot)$ is well defined and $g(t, s, \cdot)$ is continuous in $u$ (i.e., in (0.9), the $a_{i}(0)$ should be finite and the $\bar{k}_{i}$ should be continuous). The precise general assumptions are listed below. Moreover, we shall only discuss equations that are "close" to the o.d.e. case in the sense that e.g., in (0.9) var $\left(a_{i} ; 0, T\right)$ should be comparable to $a_{i}(0)$ (although not necessarily bounded by $a_{i}(0)$ ).

In the existing literature, uniqueness of solutions of $(0.6)$ is well known if $g(t, s, \cdot)$ is locally Lipschitz-continuous (see [6 and 10]). For (0.10), uniqueness is known if $\bar{k}$ is monotone nondecreasing and $a(\cdot)$ is positive, decreasing, and locally integrable [14]. A related uniqueness result for $(0.10)$ - as a consequence of a more general theory on systems of equations - has been given in [2], although with a completely different method. Estimates such as (0.2) can also be derived using the methods in [2].

Existence results that include the case of a nondifferentiable right-hand side $f$ have been given in [4] for the special structure (0.7) with $N=1$. If $f$ is, say, of bounded variation, then the existence of solutions follows for $(0.10)$ from any of the numerous a priori estimates for solutions of $(0.10)$ (see $[3,7,13])$. Of course, local existence is no problem. In the particular case where $u_{1}(t) \equiv 0$ (or more generally $u_{1}(t) \equiv C$ ) is a solution, estimates of the type (0.2) have been given under various conditions (see $[3,8,9,12,13])$, mostly in the case where $g(t, s, u)=a(t-s) \cdot \bar{k}(u)$. Here one can relax the conditions on either $a(\cdot)$ or on $\bar{k}$ considerably; it suffices to assume that $\bar{k}$ satisfies certain sign conditions and $a(\cdot)$ is merely positive definite or that $a$ is decreasing and nonnegative and that

$$
\int_{0}^{u}(\operatorname{sign} \bar{k}(r)+\operatorname{sign} r) d r
$$

as $|u| \rightarrow \infty$. The estimate corresponding to $(0.2)$ is then

$$
|u(t)| \leqslant G\left(|f(0)|+\int_{0}^{t}\left|f^{\prime}(s)\right| d s\right)
$$

with some function $G$ that can be given explicitly. With a method similar to the one employed in this note, an estimate of this type has been shown in [1] if $\bar{k}$ satisfies $\int_{0}^{u} \operatorname{sign} \bar{k}(r) d r \rightarrow \infty$ as $|u| \rightarrow \infty$ and if $a$ satisfies $a(0) \geqslant \int_{0}^{\infty}\left|a^{\prime}(s)\right| d s$.

Here is an overview of the results of this note if $g$ has the form (0.9), which may also serve as a collection of examples for our various results. Let $u, u_{1}, u_{2}$ be solutions of (0.6) corresponding to right-hand sides $f, f_{1}, f_{2}$, and assume throughout that $a_{i}(0)=1$ for all $i$.

(i) In Theorem 1.1 we prove that solutions of (0.6) with locally essentially bounded right-hand sides are unique if the $a_{i}$ are absolutely continuous on some small interval $[0, \varepsilon]$ and if the $\bar{k}_{i}$ are sums of nondecreasing and locally Lipschitz-continuous functions. If the $\bar{k}_{i}$ are instead sums of affine and nondecreasing functions, then the uniqueness assertion holds even for only measureable right-hand sides $f$, provided $u-f$ is required to be locally bounded and the $\bar{k}_{i}(u(\cdot))$ are required to be locally integrable. 
(ii) In Theorem 2.1 it is shown that the estimate (0.2) holds for $0 \leqslant t \leqslant T$ if the $\bar{k}_{i}$ are nondecreasing and if $\operatorname{var}\left(a_{i} ; 0, T\right) \leqslant a_{i}(0)=1$.

(iii) As a consequence (using a suitable transformation) we show in Corollary 2.2: If the $a_{i}$ are absolutely continuous on $[0, T]$ and the $\bar{k}_{i}$ are nondecreasing, up to affine functions, then there exists $C=C(T)$ such that

$$
\left|u_{1}(t)-u_{2}(t)\right| \leqslant C \cdot\left\{\left|f_{1}(0)-f_{2}(0)\right|+\int_{0}^{t}\left|\left(f_{1}-f_{2}\right)^{\prime}(s)\right| d s\right\} \quad \text { for } 0 \leqslant t \leqslant T .
$$

(iv) In Corollary 2.3 it is assumed that the $\bar{k}_{i}$ are nondecreasing, the $a_{i}$ are nonnegative and satisfy $a_{i}^{\prime}+c \cdot a_{i} \leqslant 0$ for some $c>0$. Then

$$
\begin{aligned}
\left|u_{1}(t)-u_{2}(t)\right| \leqslant & e^{-c t}\left|f_{1}(0)-f_{2}(0)\right| \\
& +\int_{0}^{t} e^{c \cdot(s-t)}\left|\left(f_{1}-f_{2}\right)^{\prime}+c \cdot\left(f_{1}-f_{2}\right)\right|(s) d s .
\end{aligned}
$$

(v) The assumptions of Corollary 2.4 are that $u \rightarrow \bar{k}_{i}(u)-\delta u$ is nondecreasing for some $\delta>0$, and that $\int_{0}^{\infty}\left|a_{i}^{\prime}(s)\right| e^{\delta s} d s \leqslant a_{i}(0)$. We show that there exists an $\varepsilon>0$ such that for all $t \geqslant 0$

$$
\left|u_{1}(t)-u_{2}(t)\right| \leqslant e^{-\varepsilon t}\left|f_{1}(0)-f_{2}(0)\right|+\int_{0}^{t} e^{-\varepsilon(t-s)}\left|\left(f_{1}-f_{2}\right)^{\prime}(s)\right| d s .
$$

This result reminds us of similar estimates that can easily be obtained for linear Volterra equations using Laplace transform arguments. As a consequence, if $f^{\prime}(t) \rightarrow$ $f_{\infty}$ as $t \rightarrow \infty$, then $u(t) \rightarrow u_{\infty}$.

(vi) In Corollary 2.5 it is assumed that the $a_{i}$ are positive, nonincreasing and logarithmically convex, and that the $\bar{k}_{i}$ are nondecreasing. We then show that $\left|u_{1}(t)-u_{2}(t)\right| \leqslant p(t)$, where $p$ satisfies the ordinary differential equation $p^{\prime}(t)=$ $\beta(t) p(t)+k(t), \quad p(0)=\left|f_{1}(0)-f_{2}(0)\right|, \quad \beta(t)=\max _{i} \log a_{i}^{\prime}(t)$ and $k(t)=$ $\left|\left(f_{1}-f_{2}\right)^{\prime}(t)-\beta(t)\left(f_{1}(t)-f_{2}(t)\right)\right|$. In other words, we obtain an estimate similar to the one in (iv), with the factor $e^{-\varepsilon t}$ essentially replaced by $\min a_{i}(t)$. As a consequence, some well-known and some new properties of resolvent kernels for scalar linear equations are derived.

(vii) In Theorem 3.1 and Corollary 3.2, an existence result is given: If the $\bar{k}_{i}$ are nondecreasing, up to affine functions, if the $a_{i}$ are absolutely continuous on $[0, T]$, and if $|f(\cdot)|$ and $\left|\bar{k}_{i}(f(\cdot)+C)\right|$ are integrable for all $C \in \mathbf{R}$, then a solution of $(0.6)$ on $[0, T]$ exists (which will be unique by the previous results).

In the concluding section of the paper, we give a similar outlook on the results in part II of this note [17].

Here are some of the general assumptions that will be used below: The function $g(\cdot, \cdot, u)$ is measurable for all $u \in \mathbf{R}, g(t, s, \cdot)$ is continuous for almost all $(t, s) \in \Delta$ $=\{(t, s) \mid 0 \leqslant s \leqslant t \leqslant T\}$ and for every $M>0$ there exists some $\mu_{M} \in L^{1}(\Delta, \mathbf{R})$ such that for all $|u| \leqslant M$

$$
|g(t, s, u)|+\left|\partial_{t} g(t, s, u)\right| \leqslant \mu_{M}(t, s) .
$$

If $g$ has the structure (0.7), we assume that the $k_{i}$ are measurable in $s$ for fixed $u$, continuous in $u$ for a.e. fixed $s$, and that

$$
\left|k_{i}(s, u)\right| \leqslant \delta_{M}(s)
$$


if $|u| \leqslant M$, with $\delta_{M} \in L^{1}(0, T ; \mathbf{R})$. Also, in this case the $a_{i}$ and $\partial_{t} a_{i}$ are assumed to be in $L^{1}(\Delta, \mathbf{R})$.

If $g$ has the structure (0.8), then $\partial_{s} \bar{g}(s, u)$ should be measurable in $s$ for fixed $u$, continuous in $u$ for a.e. fixed $s$, and an estimate of the form (0.11) should hold for $\partial_{s} \bar{g}(s, u)$. Also, $\bar{g}(0, \cdot)$ should be continuous.

Finally, if $g$ has the structure (0.9), then the $a_{i}$ should be absolutely continuous on $[0, T]$, and the $\bar{k}_{i}$ should be continuous. We also assume that

$$
a_{i}(0)=1 \text { for } 1 \leqslant i \leqslant N .
$$

If $(0.6)$ is considered on $[0, \infty)$, then all these assumptions should hold on any compact $t$-interval.

For $u, v \in \mathbf{R},(t, s) \in \Delta$ we abbreviate

$$
\begin{gathered}
g_{1}(t, s, u)=\partial_{t} g(t, s, u), \\
h(t, s ; u, v)=(g(t, s, u)-g(t, s, v)) \operatorname{sign}(u-v), \\
h_{1}(t, s ; u, v)=\partial_{t} h(t, s ; u, v) .
\end{gathered}
$$

In the proofs, a simple argument will be used frequently which we state as

Lemma 0. Let $q \in L^{1}(\Delta, \mathbf{R})$ such that $\partial_{t} q \in L^{1}(\Delta, \mathbf{R})$ exists, and let $p:[0, T] \rightarrow \mathbf{R}$ be measurable such that

$$
p(t)=\operatorname{sign}(q(t, t)) \text { for a.e.t. }
$$

(a) Then

$$
\int_{0}^{T} p(t) \frac{d}{d t}\left(\int_{0}^{t} q(t, s) d s\right) d t \geqslant \int_{0}^{T}\left(|q(t, t)|-\int_{0}^{T}\left|\partial_{r} q(r, t)\right| d r\right) d t .
$$

(b) If additionally for all $(t, s) \in \Delta$

$$
\begin{gathered}
p(s) \cdot q(t, s)=|q(t, s)|, \\
p(s) \cdot \partial_{t} q(t, s)=-\left|\partial_{t} q(t, s)\right|,
\end{gathered}
$$

then

$$
\int_{0}^{T} p(t) \frac{d}{d t}\left(\int_{0}^{t} q(t, s) d s\right) d t \geqslant \int_{0}^{T}|q(T, t)| d t
$$

Proof.

$$
\begin{aligned}
\int_{0}^{T} p(t) & \frac{d}{d t}\left(\int_{0}^{t} q(t, s) d s\right) d t \\
\quad= & \int_{0}^{T} p(t) \cdot\left(q(t, t)+\int_{0}^{t} \frac{\partial}{\partial t} q(t, s) d s\right) d t \\
\quad= & \int_{0}^{T}\left\{p(t) \cdot q(t, t)+\int_{t}^{T} p(s) \cdot \frac{\partial}{\partial s} q(s, t) d s\right\} d t .
\end{aligned}
$$


To prove (0.17), we estimate this by

$$
\cdots \geqslant \int_{0}^{T}\left\{|q(t, t)|-\int_{t}^{T}\left|\frac{\partial}{\partial s} q(s, t)\right| d s\right\} d t .
$$

To prove $(0.20)$, we estimate

$$
\begin{aligned}
\cdots & \geqslant \int_{0}^{T}\left\{p(t) \cdot q(t, t)+\int_{t}^{T} p(t) \cdot \frac{\partial}{\partial s} q(s, t) d s\right\} d t \\
& =\int_{0}^{T} p(t) \cdot q(T, t) d t=\int_{0}^{T}|q(T, t)| d t . \quad \text { Q.E.D. }
\end{aligned}
$$

It is a pleasure to thank Professor O. Staffans for a useful discussion on relations between some of these results and those presented in [16] and Professor J. Levin for illuminating comments, in particular for drawing the author's attention to the paper [15].

1. This section contains some general uniqueness conditions for solutions of (0.6). For the function $g$ appearing in (0.6), define $h(t, s ; u, v)$ as in (0.14).

THEOREM 1.1. Let $g$ have the general properties listed in $\S 0$.

(i) Assume that for any $M>0$ there exist $\varepsilon>0, \mu \in L^{1}(0, T ; \mathbf{R})$ such that for all numbers $u, v$ with $|u|,|v| \leqslant M$ and for all $0 \leqslant s \leqslant T$

$$
h(s, s ; u, v)+\mu(s) \cdot|u-v| \geqslant \int_{s}^{\min (s+\varepsilon, T)}\left|h_{1}(t, s ; u, v)\right| d t .
$$

Then for any essentially bounded and measurable right-hand side $f$ there exists at most one locally bounded solution $u$ of $(0.6)$ on $[0, T]$.

(ii) Assume that there exist $\varepsilon>0, \mu \in L^{1}(0, T ; \mathbf{R})$ such that (1.1) holds for all $u$, $v \in \mathbf{R}$ and all $0 \leqslant s \leqslant T$. Then for any measurable right-hand side $f$ there exists at most one solution $u(\cdot)$ of $(0.6)$, for which $u-f$ is locally bounded and for which $(t, s) \mapsto|g(t, s, u(s))|+\left|g_{1}(t, s, u(s))\right|$ is integrable.

Proof. Let $u, v$ be two solutions of $(0.6)$ on $[0, T]$, and assume that $(u-v) \equiv 0$ on $\left[0, t_{0}\right]$ for some $t_{0}$. We show that $(u-v) \equiv 0$ on some larger interval $\left[0, t_{1}\right]$.

In (i), there exists some $M>0$ such that $|u(t)|+|v(t)| \leqslant M$ for $0 \leqslant t \leqslant T$. Let $\varepsilon>0, \mu \in L^{1}(0, T ; \mathbf{R})$ be given such that (1.1) holds. Subtract the equations for $u$ and $v$, and put

$$
\begin{aligned}
p(t) & =\operatorname{sign}(u(t)-v(t)), \\
q(t, s)=g(t, s, u(s)) & -g(t, s, v(s))+\mu(s) \cdot(u(s)-v(s)) .
\end{aligned}
$$

Thus for $t_{0} \leqslant t \leqslant t_{0}+\varepsilon=t_{1}$,

$$
u(t)-v(t)+\int_{t_{0}}^{t} q(t, s) d s=\int_{t_{0}}^{t} \mu(s) \cdot(u(s)-v(s)) d s .
$$

The assumptions for $g$ imply that $(u-v)(\cdot)$ is absolutely continuous; thus differentiate (1.4), multiply with $p(t)$, and integrate from $t_{0}$ to $\tau \leqslant t_{1}$. Using (0.17), this gives

$$
\begin{gathered}
|u(\tau)-v(\tau)|+\int_{t_{0}}^{\tau} h(s, s ; u(s), v(s)) d s \\
\quad \leqslant \int_{t_{0}}^{\tau} \int_{s}^{\tau}\left|h_{1}(\sigma, s ; u(s), v(s))\right| d \sigma d s
\end{gathered}
$$


which implies by assumption (1.1)

$$
|u(\tau)-v(\tau)| \leqslant \int_{t_{0}}^{\tau} \mu(s) \cdot|u(s)-v(s)| d s .
$$

Gronwall's lemma [19] then implies that $u \equiv v$ on $\left[t_{0}, t_{1}\right]$. By a standard argument, $u \equiv v$ on $[0, T]$ follows.

To prove uniqueness in (ii), we proceed in the same way, the only change being that $\varepsilon$ and $\mu(\cdot)$ are chosen independently of any bounds on $u$ and $v$. Q.E.D.

If $g$ has the structure

$$
g(t, s, u)=\sum_{i=1}^{N} a_{i}(t, s) k_{i}(s, u),
$$

then a uniqueness result follows by checking the assumption of the theorem above:

Corollary 1.2. (i) Assume that for any $M>0$ there exists $\mu \in L^{1}(0, T ; \mathbf{R})$ such that $u \rightarrow k_{i}(s, u)+\mu(s) u$ is nondecreasing on $[-M, M]$ for all $i$ and almost all s. Also, assume that there are $K, \varepsilon>0$ such that for all $i$ and almost all $0 \leqslant s \leqslant T$

$$
\int_{s}^{\min (s+\varepsilon, T)}\left|\partial_{t} a_{i}(t, s)\right| d t \leqslant a_{i}(s, s) \leqslant K .
$$

Then the uniqueness conclusion in Theorem 1.1(i) holds.

(ii) Assume that the $k_{i}$ and $a_{i}$ satisfy the properties stated in (i) with $\mu(\cdot)$ independent of $M$. Then the uniqueness conclusion of Theorem 1.1(ii) is true.

One might ask if the uniqueness assertion in (i) of the above theorem remains valid if unbounded right-hand sides $f(\cdot)$ are also allowed. That this is not the case can be seen from the following

CountereXample. Let $g_{0}:[0,1] \times \mathbf{R} \rightarrow \mathbf{R}$ be defined by

$$
\begin{gathered}
g_{0}(0, u)=0 \text { for all } u, \\
g_{0}(t, u)=y \Leftrightarrow y^{3}+\frac{5}{9} \cdot t \cdot y=u, \quad \text { if } 0<t \leqslant 1, u \in \mathbf{R},
\end{gathered}
$$

and let $r:(0,1) \rightarrow \mathbf{R}$ be any integrable function for which

$$
\liminf |r(t)|=\infty \quad \text { as } t \rightarrow 0 .
$$

Define $g(t, s, u):=-g_{0}(s, u-r(s))$. Then $g$ does not depend on $t$ (the integral equation below will be equivalent to an ordinary differential equation), and $g$ is globally Lipschitz-continuous in $u$, uniformly on any interval $\left[t_{0}, 1\right]$ with $t_{0}>0$, and also locally Lipschitz-continuous in $u$, uniformly on $[0,1]$; thus it is clear that the assumptions of Theorem 1.1(i) hold for this $g$. Consequently, for any bounded measurable right-hand side $f$ the equation

$$
u(t)+\int_{0}^{t} g(t, s, u(s)) d s=f(t)
$$

has at most one solution $u$ for which $u-f$ is absolutely continuous (and, in fact, this solution will exist for any bounded measurable $f$; see the arguments in §3). But one checks easily that for the unbounded right-hand side $f(t)=r(t)$, we have the solutions $u(t)=r(t), v(t)=r(t)+\frac{2}{9} t^{3 / 2}, \quad w(t)=r(t)-\frac{2}{9} t^{3 / 2}$. To guarantee 
uniqueness for general unbounded right-hand sides, one therefore needs monotonicity and/or a global Lipschitz condition, as expressed in the conditions of Theorem 1.1; this phenomenon does not occur for ordinary differential equations (which would correspond to right-hand sides with bounded variation).

A uniqueness result related to the one stated above has been given in [15] for solutions of

$$
q(u(t), t)+\int_{0}^{t} \sum_{i} b_{i}(t, s) g_{i}(t, s, u(s)) d s=f(t),
$$

assuming comparable regularity properties for $b_{i}, g_{i}$ and $f$ (Lipschitz continuity of $g_{i}$ in $t$, a slightly weaker assumption for $\partial_{t} b_{i}(t, s)$, and $f$ should be continuous and have bounded variation), but permitting functions $q$ that are merely strictly increasing in $u$. Under the regularity assumptions used in this paper, the additional assumptions of [15] are

$$
\begin{gathered}
u \rightarrow g_{i}(t, s, u) \text { is nondecreasing, } \\
u \rightarrow \partial_{t} g_{i}(t, s, u) \text { is nonincreasing, } \\
t \rightarrow b_{i}(t, s) \text { is nonincreasing and nonnegative, }
\end{gathered}
$$

and these conditions should hold on sets $\{s \mid \max (0, t-\varepsilon) \leqslant s \leqslant t<\infty\}$ with $\varepsilon>0$. In the notation $(0.14),(0.15)$, these conditions mean

$$
h_{1}(t, s ; u, v) \leqslant 0 \leqslant h(t, s ; u, v) .
$$

This implies that (1.1) holds with $\mu=0$, and we therefore have uniqueness even for arbitrary measurable $f(\cdot)$ if $q(u, t)=u$. It is not clear, however, how to extend the proof of Theorem 1.1 to equations of the form (1.9), except if $q$ is (at least) locally Lipschitz-continuous in both variables.

In [14], a remarkable result is given for the convolution equation with one nonlinearity $(0.10)$, which asserts uniqueness also for unbounded positive decreasing kernels $a(\cdot)$ and increasing nonlinearities $\bar{k}$. This situation obviously is excluded in the assumptions of Theorem 1.1. The uniqueness question for nonconvolution equations (with one monotone nonlinearity) or equations with several monotone nonlinearities and singular kernels therefore remains open, except if one adds the ad hoc assumption that all nonlinearities are also (locally or globally) Lipschitz-continuous.

2. Next, conditions are given under which estimates of the type (0.2) can be derived. We start with a simple result.

THEOREM 2.1. Let $g$ satisfy the assumptions of $\S 0$ and assume that for all $u, v \in \mathbf{R}$, $0 \leqslant s \leqslant T$

$$
h(s, s ; u, v) \geqslant \int_{s}^{T}\left|h_{1}(t, s ; u, v)\right| d t .
$$

If $u_{1}, u_{2}$ are two solutions of $(0.6)$ with right-hand sides $f_{1}, f_{2}$ for which $f_{1}-f_{2}$ is absolutely continuous, then for all $0 \leqslant t \leqslant T$

$$
\left|u_{1}(t)-u_{2}(t)\right| \leqslant\left|f_{1}(0)-f_{2}(0)\right|+\int_{0}^{t}\left|\left(f_{1}-f_{2}\right)^{\prime}(s)\right| d s .
$$


Proof. We take the difference of $u_{1}(\cdot)$ and $u_{2}(\cdot)$ and differentiate the result. Multiplication with $\operatorname{sign}\left(u_{1}(t)-u_{2}(t)\right)=p(t)$ and integration over $[0, t]$ gives the estimate

$$
\begin{aligned}
\mid u_{1}(t) & -u_{2}(t) \mid+\int_{0}^{t} p(s) \frac{d}{d s}\left(\int_{0}^{s} q(s, r) d r\right) d s \\
= & \left|f_{1}(0)-f_{2}(0)\right|+\int_{0}^{t}\left(f_{1}-f_{2}\right)^{\prime}(s) \cdot p(s) d s
\end{aligned}
$$

with $q(s, r)=g\left(s, r, u_{1}(r)\right)-g\left(s, r, u_{2}(r)\right)$. Then (2.1) and (0.17) imply that the integral on the left-hand side of (2.3) is nonnegative, and (2.2) follows. Q.E.D.

By transforming $(0.6)$, we next deduce some consequences.

COROLlaRY 2.2. Let $g$ have the structure (0.7), and assume that

(i) for all $i, s \mapsto\left|a_{i}(s, s)\right|$ is essentially bounded,

(ii) for all $i$, all measurable sets $E \subset[0, T]$, and all $0 \leqslant s \leqslant T$

$$
\int_{[s, T] \cap E}\left|\partial_{t} a_{i}(t, s)\right| d t \leqslant \delta(|E|) \cdot a_{i}(s, s),
$$

where $|E|$ denotes the Lebesgue measure of $E$ and $\delta(h)=o(1)$ as $h \rightarrow 0$,

(iii) there exists $\mu \in L^{1}(0, T ; \mathbf{R})$ such that for all $i$ and a.e. $s$

$$
u \mapsto k_{i}(s, u)+\mu(s) \cdot u
$$

is nondecreasing.

Then there exists $K>0$, depending on $\delta(\cdot)$, on $\mu$ and on $T$, such that for any two solutions $u_{1}, u_{2}$ of $(0.6)$ with right-hand sides $f_{1}, f_{2}$

$$
\left|u_{1}(t)-u_{2}(t)\right| \leqslant K \cdot\left(\left|f_{1}(0)-f_{2}(0)\right|+\int_{0}^{t}\left|\left(f_{1}-f_{2}\right)^{\prime}(s)\right| d s\right)
$$

for all $0 \leqslant t \leqslant T$, if the right-hand side is finite.

Proof. Multiply the equation (0.6) with $L \cdot e^{L \cdot(i-t)}$, integrate from 0 to $\bar{t}$, add the result to $(0.6)$ and define $w_{j}(t)=e^{-L t} \cdot u_{j}(t)$. The constant $L>0$ will be specified later in the proof. Then $w_{j}$ satisfies

$$
\begin{aligned}
w_{j}(t) & +L \cdot \int_{0}^{t} w_{j}(s) d s+\sum_{i=1}^{N} \int_{0}^{t} \tilde{a}_{i}(t, s) \tilde{k}_{i}\left(s, w_{j}(s)\right) d s \\
& =\tilde{f}_{j}(t)+\sum_{i=1}^{N} \int_{0}^{t} \tilde{a}_{i}(t, s) e^{L s} \mu(s) w_{j}(s) d s,
\end{aligned}
$$

with

$$
\begin{gathered}
\tilde{a}_{i}(t, s)=e^{-L s} a_{i}(s, s)+\int_{s}^{t} e^{-L r} \frac{\partial}{\partial r} a_{i}(r, s) d r, \\
\tilde{k}_{i}(s, w)=k_{i}\left(s, e^{L s} \cdot w\right)+\mu(s) \cdot e^{L s} w, \\
\tilde{f}_{j}(t)=f_{j}(0)+\int_{0}^{t} e^{-L s} f_{j}^{\prime}(s) d s .
\end{gathered}
$$


Choose $L>0$ big enough such that for a.e. $s$ and all $i$

$$
a_{i}(s, s) \geqslant \int_{s}^{T} e^{-L(r-s)}\left|\frac{\partial}{\partial r} a_{i}(r, s)\right| d r,
$$

using assumption (ii). Thus

$$
\tilde{a}_{i}(s, s) \geqslant \int_{s}^{T}\left|\frac{\partial}{\partial r} \tilde{a}_{i}(r, s)\right| d r
$$

for all $i$ and a.e. $s$. Thus we can apply Theorem 2.1 to (2.5), and (2.2) implies

$$
\begin{aligned}
& \left|w_{1}(t)-w_{2}(t)\right| \leqslant\left|f_{1}(0)-f_{2}(0)\right|+\int_{0}^{t}\left|\left(\tilde{f}_{1}-\tilde{f}_{2}\right)^{\prime}(s)\right| d s \\
& \quad+\sum_{i=1}^{N} \int_{0}^{t}\left\{\tilde{a}_{i}(s, s)+\int_{s}^{t}\left|\frac{\partial}{\partial r} \tilde{a}_{i_{i}}(r, s)\right| d r\right\} e^{L s} \mu(s)\left|w_{1}(s)-w_{2}(s)\right| d s .
\end{aligned}
$$

Using Gronwall's lemma and recalling the definition of the $w_{j}$ then proves (2.4). Q.E.D.

In a similar manner, we get

COROLlaRY 2.3. Let $g$ have the structure (0.8), and assume that $\bar{g}$ satisfies

(i) $\bar{g}(t, \cdot)$ is nondecreasing for all $t$,

(ii) for some $\alpha \in \mathbf{R}, \bar{g}_{1}(t, \cdot)+\alpha \cdot \bar{g}(t, \cdot)$ is nonincreasing for all $t \in\left[0, T^{\prime}\right]$.

If $u_{1}, u_{2}$ are two solutions of $(0.6)$ with $f_{1}-f_{2}$ absolutely continuous, then for all $t \in[0, T]$

$$
\begin{aligned}
\left|u_{1}(t)-u_{2}(t)\right| \leqslant & e^{-\alpha t}\left|f_{1}(0)-f_{2}(0)\right| \\
& +\int_{0}^{t} e^{\alpha(s-t)}\left|\left(f_{1}-f_{2}\right)^{\prime}(s)+\alpha \cdot\left(f_{1}-f_{2}\right)(s)\right| d s .
\end{aligned}
$$

The proof uses the transformation $w_{j}(t)=e^{\alpha t} \cdot u_{j}(t)$, such that $w_{j}$ satisfies $(0.6)$ with $g(t, s, u(s))$ replaced by $\tilde{g}\left(t, s, w_{j}(s)\right)=e^{\alpha t} \bar{g}\left(t-s, e^{-\alpha s} w_{j}(s)\right)$ and with $f_{j}(t)$ replaced by $f_{j}(t) \cdot e^{\alpha t}$. A straightforward application of Theorem 2.1 then implies (2.12).

This estimate implies for $\alpha>0$ that $u_{1}(\cdot)$ and $u_{2}(\cdot)$ will both have the same limit (or neither will have a limit) if $\left|\left(f_{1}-f_{2}\right)(t)\right|+\left|\left(f_{1}-f_{2}\right)^{\prime}(t)\right| \rightarrow 0$ for $t \rightarrow \infty$. If $\bar{g}(t, 0)=0$ for all $t$ and $\alpha>0$, then any solution of $(0.6)$ with $|f(t)|+\left|f^{\prime}(t)\right| \leqslant K$ for all $t$ will be uniformly bounded on $[0, \infty)$. Any ordinary differential equation

$$
u^{\prime}(t)+\alpha \cdot u(t)+k(u(t))=f^{\prime}(t)+\alpha \cdot f(t)
$$

is equivalent to an integral equation for which Corollary 2.3 can be applied.

We next give a result that allows us to estimate the difference of two solutions in terms of $\left(f_{1}-f_{2}\right)^{\prime}$ alone by a similar exponential expression.

Corollary 2.4. Assume that $g$ has the form (0.9) and that there exists $\delta>0$ such that for all $i$

(i) $u \mapsto \bar{k}_{i}(u)-\delta \cdot u$ is nondecreasing,

(ii) $\int_{0}^{\infty}\left|a_{i}^{\prime}(s)\right| \cdot e^{\delta s} d s \leqslant a_{i}(0)=1$. 
Then there exists $\varepsilon>0$ such that for any two solutions $u_{1}, u_{2}$ of $(0.6)$ with $f_{1}-f_{2}$ absolutely continuous

$$
\left|u_{1}(t)-u_{2}(t)\right| \leqslant e^{-\varepsilon t}\left|f_{1}(0)-f_{2}(0)\right|+\int_{0}^{t} e^{-\varepsilon(t-s)}\left|\left(f_{1}-f_{2}\right)^{\prime}(s)\right| d s
$$

for all $t \geqslant 0$.

As a consequence of this estimate, for any solution $u(\cdot)$ of $(0.6)$ for which $f^{\prime}(t) \rightarrow \alpha(t \rightarrow \infty)$ we have $u(t) \rightarrow u_{\infty}$ as $t \rightarrow \infty$, and $u_{\infty}$ is the unique number for which

$$
\sum_{i=1}^{N} a_{i}(\infty) \bar{k}_{i}\left(u_{\infty}\right)=\alpha
$$

Indeed, assumptions (i) and (ii), respectively, imply that $a_{i}(\infty)>0$ and $\bar{k}_{i}: \mathbf{R} \rightarrow \mathbf{R}$ is one-to-one, thus (2.15) has a solution $u_{\infty}$. Now $u_{\infty}$ solves (0.6) with right-hand side

$$
\tilde{f}(t)=t \cdot \alpha+\sum_{i=1}^{N} \int_{0}^{t}\left(a_{i}(s)-a_{i}(\infty)\right) d s \cdot \bar{k}_{i}\left(u_{\infty}\right)+u_{\infty},
$$

thus

$$
\tilde{f}^{\prime}(t)=\alpha+\sum_{i=1}^{N}\left(a_{i}(t)-a_{i}(\infty)\right) \bar{k}_{i}\left(u_{\infty}\right) .
$$

Comparing $u(\cdot)$ to $u_{\infty}$ gives

$$
\begin{aligned}
\left|u(t)-u_{\infty}\right| \leqslant & e^{-\varepsilon t}\left|f(0)-u_{\infty}\right| \\
& +\int_{0}^{t} e^{\varepsilon(s-t)} \cdot\left|f^{\prime}(s)-\alpha-\sum_{i=1}^{N}\left(a_{i}(s)-a_{i}(\infty)\right) \bar{k}_{i}\left(u_{\infty}\right)\right| d s
\end{aligned}
$$

and thus $u(t) \rightarrow u_{\infty}$ as $t \rightarrow \infty$.

Proof of Corollary 2.4. Taking the convolutions of $(0.6)$ with $-\varepsilon \cdot e^{-\varepsilon t}$ and adding the result to $(0.6)$ gives a new equation for $v_{j}(t)=e^{\varepsilon t} \cdot u_{j}(t)$, namely

$$
\begin{aligned}
v_{j}(t) & +\sum_{i=1}^{N} \int_{0}^{t} \tilde{a}_{i}(t-s) \tilde{k}_{i}\left(s, v_{j}(s)\right) d s \\
& +\int_{0}^{t} \sum_{i=1}^{N}\left(\delta \cdot \tilde{a}_{i}(t-s)-\varepsilon\right) \cdot v_{j}(s) d s=\tilde{f}_{j}(t),
\end{aligned}
$$

with (cf. the proof of Corollary 2.2)

$$
\begin{gathered}
\tilde{a}_{i}(t)=1+\int_{0}^{t} e^{\varepsilon s} a_{i}^{\prime}(s) d s, \\
\tilde{k}_{i}(s, v)=e^{\varepsilon s} \cdot \bar{k}_{i}\left(e^{-\varepsilon s} v\right)-\delta \cdot v, \\
\tilde{f}_{j}(t)=f_{j}(0)+\int_{0}^{t} e^{\varepsilon s} f_{j}^{\prime}(s) d s .
\end{gathered}
$$


Fix $\varepsilon>0$ small enough such that for all $i$

$$
a_{i}(0)-\int_{0}^{\infty} e^{\varepsilon s}\left|a_{i}^{\prime}(s)\right| d s \geqslant \frac{\varepsilon}{\delta}
$$

and $\varepsilon<\delta$. Then the integral operator on the left-hand side of (2.16) satisfies the assumptions of Theorem 2.1, and the conclusion (2.14) follows from (2.2), recalling the definition of $v_{j}$. Q.E.D.

If $g$ has the structure (0.9) and if the kernels $a_{i}$ are log-convex, then the factor $e^{-\varepsilon t}$ in (2.12) can be replaced by essentially $\min _{i} a_{i}(t)$. More generally:

COROLlary 2.5. Let $g$ have the structure (0.7), let the $a_{i}$ be positive, and assume that $\partial_{t} a_{i}(t, s) \leqslant 0$ for a.e. $(t, s) \in \Delta$ and that

$$
0 \geqslant \partial_{t} \partial_{s} \log a_{i} \in L^{1}(\Delta)
$$

for all $i$. Also, assume that $u \mapsto k_{i}(s, u)$ is nondecreasing for a.e. s. Define

$$
\beta(t)=\max _{i} \frac{\partial_{t} a_{i}(t, 0)}{a_{i}(t, 0)} .
$$

If $u_{1}, u_{2}$ are two solutions of $(0.6)$ with right-hand sides $f_{1}, f_{2}$, let

$$
k(t)=\left|\left(f_{1}-f_{2}\right)^{\prime}(t)-\beta(t) \cdot\left(f_{1}-f_{2}\right)(t)\right| .
$$

Then

$$
\left|u_{1}(t)-u_{2}(t)\right| \leqslant p(t) \text { for all } t \geqslant 0,
$$

where

$$
p^{\prime}(t)=\beta(t) \cdot p(t)+k(t), \quad p(0)=\left|f_{1}(0)-f_{2}(0)\right| .
$$

In other words,

$$
\begin{aligned}
\left|u_{1}(t)-u_{2}(t)\right| \leqslant b(t) \cdot & || f_{1}(0)-f_{2}(0) \mid \\
& \left.+\int_{0}^{t} \frac{1}{b(s)}\left|\left(f_{1}-f_{2}\right)^{\prime}(s)-\beta(s)\left(f_{1}-f_{2}\right)(s)\right| d s\right)
\end{aligned}
$$

with

$$
b(t)=\exp \left(\int_{0}^{t} \beta(s) d s\right) .
$$

Proof. Define $v_{j}(t)=(b(t))^{-1} \cdot u_{j}(t)$. Then

$$
v_{j}(t)+\sum_{i=1}^{N} \int_{0}^{t} \frac{a_{i}(t, s)}{b(t)} k_{i}\left(s, b(s) \cdot v_{j}(s)\right) d s=\frac{f_{j}(t)}{b(t)} .
$$

Now $v \mapsto k_{i}(s, b(s) \cdot v)$ is nondecreasing, and (2.21) and the definition of $b$ imply

$$
\begin{aligned}
\int_{s}^{T}\left|\frac{\partial}{\partial t} \frac{a_{i}(t, s)}{b(t)}\right| d t & =-\int_{s}^{T} \frac{\partial_{t} a_{i}(t, s) b(t)-a_{i}(t, s) \partial_{t} b(t)}{b^{2}(t)} d t \\
& =\frac{a_{i}(s, s)}{b(s)}-\frac{a_{i}(T, s)}{b(T)} \leqslant \frac{a_{i}(s, s)}{b(s)} .
\end{aligned}
$$


Thus we can apply Theorem 2.1 to get (2.26) or equivalently (2.24) and (2.25). Q.E.D.

In the special case of the equation

$$
r(t, s)+\int_{s}^{t} a(t, \tau) r(\tau, s) d \tau=a(t, s) \text { for } t \geqslant s
$$

for a kernel $a$ satisfying the assumptions of Corollary 2.5, we get some well-known results on $r(t, s)$, the resolvent of $a(t, s)$ (cf. [10]).

(i) In this situation, for fixed $s$, one obtains $b(t)=a(t, s)$ in (2.27). Then (2.26) implies that

$$
|r(t, s)| \leqslant a(t, s) \quad \text { for } t \geqslant s .
$$

It is well known that in this situation in fact $r(t, s) \geqslant 0$ (cf. also [17]).

(ii) Assume that $\partial a(t, s) /\left.\partial s\right|_{t=s}$ is finite and nonnegative for all $s \geqslant 0$. Then the derivative $r_{2}(t, s)=\partial r(t, s) / \partial s$ satisfies (2.30)

$$
r_{2}(t, s)+\int_{s}^{t} a(t, \tau) r_{2}(\tau, s) d \tau=a(t, s) \cdot r(s, s)+\frac{\partial}{\partial s} a(t, s) \text { for } t \geqslant s .
$$

A straightforward application of (2.26) then gives the estimate

$$
\left|r_{2}(t, s)\right| \leqslant a(t, s) \cdot\left(\frac{a_{2}(s)}{a_{1}(s)}+a_{1}^{2}(s)+a_{2}(s)\right)-\frac{\partial}{\partial s} a(t, s),
$$

with the abbreviations $a_{1}(s)=a(s, s)$ and $a_{2}(s)=\partial a(t, s) /\left.\partial s\right|_{t=s}$. It will be shown in [17] that under these conditions actually $r_{2}(t, s) \geqslant 0$.

(iii) It is not clear how to derive an estimate for $\partial r(t, s) / \partial t$ in this way.

In [15], an estimate of the form (2.2) was given for solutions of (1.9) under the assumptions listed in (1.10)-(1.13). There it was also noted that it suffices to show (2.2) for $u_{2} \equiv 0$, since the difference of two solutions of (0.6) will again satisfy an equation of this form.

3. In this section we give conditions under which (0.6) will have a solution. We treat the general case of nondifferentiable $f(\cdot)$. Under the conditions given the solution will be unique. For simplicity, in this section let

$$
g(t, s, 0)=0 \text { for } 0 \leqslant s \leqslant t \leqslant T .
$$

The first result gives conditions that guarantee the existence of solutions on small time intervals.

THEOREM 3.1. Assume that $g$ has the properties listed in $\S 0$ and that there exists a $\mu(\cdot) \in L^{1}\left(0, T ; \mathbf{R}^{+}\right)$such that for a.e. $0 \leqslant s \leqslant T$ and all $u, v$

$$
\begin{gathered}
h(s, s ; u, v)+\mu(s) \cdot|u-v| \geqslant \int_{s}^{T}\left|h_{1}(t, s ; u, v)\right| d t, \\
\int_{0}^{T} \mu(s) \cdot|f(s)| d s<\infty, \\
\int_{0}^{T}|g(s, s, f(s)+K)| d s<\infty \text { for all } K \in \mathbf{R} .
\end{gathered}
$$

Then (0.6) has exactly one solution $u(\cdot)$ such that $u(\cdot)-f(\cdot)$ is absolutely continuous on $[0, T]$. 
Proof. For $M>0$, let $p_{M}(v)=\min (-M, \max (v, M))$. Then define for $0 \leqslant \sigma \leqslant 1$ and $M>0$ the operator

$$
F_{M}^{\sigma}(v)(t)=-\sigma \cdot \int_{0}^{t} g\left(t, s, p_{M}(v(s))+f(s)\right) d s
$$

if $v \in L^{p}(0, T ; \mathbf{R}), p<\infty$ fixed. We list some properties of $F_{M}^{\sigma}$ :

(i) $F_{M}^{o}$ has values in a bounded set in $L^{\infty}(0, T ; \mathbf{R})$. From (3.2) and (3.4) it follows that for $-M \leqslant v(s) \leqslant M$

$$
\begin{gathered}
g(s, s, f(s)-M)+\mu(s) \cdot(-M-v(s)) \leqslant g(s, s, v(s)+f(s)) \\
\leqslant g(s, s, f(s)+M)+\mu(s) \cdot(M-v(s))
\end{gathered}
$$

and thus for $0 \leqslant s \leqslant t \leqslant T$

$$
\begin{aligned}
|g(t, s, f(s)+v(s))| \leqslant & |g(s, s, f(s)+v(s))| \\
& +\int_{s}^{t}\left|g_{1}(\tau, s, f(s)+v(s))\right| d \tau \\
\leqslant & 2 \cdot|g(s, s, f(s)+v(s))|+\mu(s) \cdot|f(s)+v(s)|,
\end{aligned}
$$

thus $\left|F_{M}^{\sigma}(v)(t)\right| \leqslant C_{M}$ for a.e. $t$.

(ii) $F_{M}^{\sigma}$ is continuous from $L^{p}(0, T ; \mathbf{R})$ into $L^{p}(0, T ; \mathbf{R})$. This follows from the Carathéodory-conditions that we assumed in $\S 0$.

(iii) $F_{M}^{\sigma}$ has values in a bounded set in $W^{1,1}([0, T], \mathbf{R})$. To show this, notice that $F_{M}^{o}(v)(\cdot)$ is differentiable a.e., and

$$
\begin{aligned}
& \int_{0}^{T}\left|\frac{d}{d t} F_{M}^{o}(v)(t)\right| d t \leqslant \int_{0}^{T}(2 \cdot \mid g\left(t, t, f(t)+p_{M}(v(t))\right) \mid \\
&\left.+\mu(t) \cdot\left|p_{M}(v(t))+f(t)\right|\right) d t,
\end{aligned}
$$

and the argument of (i) shows that this integral is bounded.

(iv) Thus $F_{M}^{\sigma}: L^{p}(0, T ; \mathbf{R}) \rightarrow L^{p}(0, T ; \mathbf{R})$ is completely continuous.

(v) Any solution $\tilde{v}$ of $\tilde{v}(\cdot)=F_{M}^{o}(\tilde{v})(\cdot)$ on $[0, T]$ satisfies an estimate

$$
\text { ess sup }|\tilde{v}(t)| \leqslant C^{*},
$$

where the constant $C^{*}$ is independent of $M$. This follows by comparing $\tilde{v}$ with $w \equiv 0$, which is a solution of

$$
w(t)=F_{M}^{o}(w)(t)+\sigma \int_{0}^{t} g(t, s, f(s)) d s .
$$

In a manner that is analogous to the proof of Theorem 1.1, and using (3.2), we get

$$
\begin{aligned}
|\tilde{v}(t)| & =|\tilde{v}(t)-w(t)| \\
& \leqslant \int_{0}^{t}|\tilde{v}(s)| \cdot \mu(s) d s+\int_{0}^{t}\left|\frac{d}{d s} \int_{0}^{s} g(s, \tau, f(\tau)) d \tau\right| d s \\
& \leqslant \tilde{C}+\int_{0}^{t} \mu(s)|\tilde{v}(s)| d s,
\end{aligned}
$$

and Gronwall's lemma implies the desired result. 
We now apply a standard degree argument to the family of mappings $\left(F_{C^{*}+1}^{\sigma}\right)_{0 \leqslant \sigma \leqslant 1}$ on a sufficiently large ball about the origin in $L^{p}(0, T ; \mathbf{R})$. Since $F_{M}^{0}$ has a unique fixed point (namely 0 ), $F_{C^{*}+1}^{1}$ will have at least one fixed point $v^{*}[11]$. By (v), $\left\|v^{*}\right\|_{L^{\infty}} \leqslant C^{*}$, thus $v^{*}$ is in fact a solution of

$$
v^{*}(t)+\int_{0}^{t} g\left(t, s, f(s)+v^{*}(s)\right) d s=0 .
$$

Putting $u(t)=f(t)+v^{*}(t)$ we obtain a solution of (0.6). Theorem 1.1 implies that $u$ must be unique if $u-f$ is to be essentially bounded. Q.E.D.

Property (3.2) is true if $g$ has the structure (0.7), if the $a_{i}$ satisfy

$$
\int_{s}^{T}\left|\partial_{t} a_{i}(t, s)\right| d t \leqslant a_{i}(s, s) \leqslant K
$$

for a.e. $s$ and if the $k_{i}$ satisfy

$$
u \rightarrow k_{i}(s, u)+\mu(s) \cdot u
$$

is nondecreasing for some $\mu$. In particular, if $g$ has the structure (0.9), if the $a_{i}$ are absolutely continuous and if the $\bar{k}_{i}$ are nondecreasing (up to linear functions), then (3.2) always holds on some small interval that can be fixed a priori. A standard translation-induction argument then allows to continue the solution, if the data permit this.

COROllary 3.2. Let $g, f$ be as in Theorem 3.1, with the exception that in (3.2) $T$ is replaced by $\min (s+\varepsilon, T)$ for some $\varepsilon>0$. Also assume that for a.e. $0 \leqslant s \leqslant t \leqslant T$ and all $u \in \mathbf{R}$

$$
|g(t, s, u)| \leqslant K \cdot|g(s, s, u)|+b(s)
$$

with some $K>0$ and $b \in L^{1}(0, T ; \mathbf{R})$. Then $(0.6)$ has a unique solution $u(\cdot)$ on $[0, T]$ for which $u-f$ is absolutely continuous.

To prove this corollary, assume that the solution has already been constructed on some $\left[0, T_{1}\right]$ (by Theorem 3.1) and rewrite $(0.6)$ on $\left[T_{1}, T_{1}+\varepsilon\right]$ as

$$
u(t)+\int_{T_{1}}^{t} g(t, s, u(s)) d s=-\int_{0}^{T_{1}} g(t, s, u(s)) d s+f(t) .
$$

(3.6) and (3.7) show that

$$
\int_{0}^{T_{1}}|g(t, s, u(s))| d s \leqslant K .
$$

Thus an application of Theorem 3.1 implies that the solution has a unique continuation on $\left[0, T_{1}+\varepsilon\right]$. Q.E.D.

From these arguments it also follows that (3.13) can be omitted from the assumptions if $f$ is essentially bounded. It is easy to set up conditions that guarantee a function $g$ of the separable structure $(0.7)$ or $(0.9)$ to meet the assumptions of Corollary 3.2: If $g$ has the form (0.9), it is sufficient to assume that the $a_{i}$ are in $L^{1}(0, T)$. Also, $u \rightarrow \bar{k}_{i}(u)+\mu \cdot u$ should be nondecreasing for some nonnegative $\mu$, and $s \rightarrow \mu \cdot|f(s)|+\sum_{i}\left|\bar{k}_{i}(f(s)+K)\right|$ should be integrable for all real numbers $K$. 
For equations of the form (0.7), conditions similar to (3.11) and (3.12) have to hold. In particular, if the $\bar{k}_{i}$ are strongly growing, then this condition gives a quite precise statement about the admissible forcing function $f(\cdot)$; on the other hand, if the $\bar{k}_{i}$ are nondecreasing $(\mu=0)$, but only slowly growing, then also nonintegrable right-hand sides $f$ can be admitted.

As an example, consider the equation with one nonlinearity

$$
u(t)+\int_{0}^{t} a(t-s) \bar{k}(u(s)) d s=f(t)
$$

with some absolutely continuous kernel $a(\cdot), a(0)=1$. If $\bar{k}(u)=\sinh u$, then solutions exist and are unique for any measurable right-hand side $f$ for which

$$
\int_{0}^{T}|\exp | f(s)|| d s<\infty .
$$

If $\bar{k}(u)=\log (\max [1, u])$, then solutions exist, and are unique for any measurable right-hand side $f$ for which

$$
\int_{0}^{T} \log (\max \{1, f(s)\}) d s<\infty .
$$

These examples suggest that an $L^{p}$-setting for scalar equations of the form (0.6) or even $(0.10)$ is not always adequate. It is not clear, however, how to extend the results given here to systems of equations. For a different approach to equations of the form (0.10) that works also for systems and that gives existence results for $f \in$ $L^{p}(0, T)$, see [4 and 5].

Conclusion and outlook. The scalar Volterra integral equation

$$
u(t)+\int_{0}^{t} g(t, s, u(s)) d s=f(t)
$$

has been studied, and conditions for $g$ have been given such that for two solutions $u_{1}, u_{2}$ of (0.6) with right-hand sides $f_{1}, f_{2}$

$$
\left\|u_{1}-u_{2}\right\|_{L^{\infty}(0, T ; \mathbf{R})} \leqslant\left|f_{1}(0)-f_{2}(0)\right|+\left\|\left(f_{1}-f_{2}\right)^{\prime}\right\|_{L^{1}(0, T ; \mathbf{R})} .
$$

In the case where $g(t, s, u)=\sum_{i \leqslant N} a_{i}(t-s) \bar{k}_{i}(u)$, these conditions reduce to

$$
\bar{k}_{i}(\cdot) \text { is nondecreasing, } \operatorname{var}\left(a_{i} ; 0, T\right) \leqslant a_{i}(0) .
$$

Various transformations have been employed to give versions of $(*)$ with weight factors on the right-hand side, thus displaying the Lipschitz character of the solution operators for (0.6) from $W^{1,1}([0, T], \mathbf{R})$ (equipped with various norms) into $L^{\infty}(0, T ; \mathbf{R})$. The case $T=\infty$ is included. As a consequence, uniqueness and existence results have been derived.

In part II of this note [17], it will be shown that under closely related conditions the solution operators for $(0.6)$ are actually Lipschitz continuous from $W^{1,1}([0, T], \mathbf{R})$ into itself. More specifically, if $g(t, s, u)$ has the special form above and $a_{i}(0)>0$, $\operatorname{var}\left(a_{i} ; 0, T\right)<\infty$, the following results will be shown $\left(u, u_{1}, u_{2}\right.$ are solutions corresponding to $\left.f, f_{1}, f_{2}\right)$ :

(i) If the $\bar{k}_{i}$ are nondecreasing and $\operatorname{var}\left(a_{i} ; 0, T\right) \leqslant a_{i}(0)-c$ for some $c>0$, then

$$
\int_{0}^{T}\left|\left(u_{1}-u_{2}\right)^{\prime}(t)\right| d t \leqslant\left(2 c^{-1}-1\right)\left|f_{1}(0)-f_{2}(0)\right|+2 c^{-1} \int_{0}^{T}\left|\left(f_{1}-f_{2}\right)^{\prime}(t)\right| d t \text {. }
$$


(ii) If the $\bar{k}_{i}$ are nondecreasing, then $\operatorname{var}\left(f^{\prime} ; 0, T\right)<\infty$ implies $\operatorname{var}\left(u^{\prime} ; 0, T\right)<\infty$.

(iii) If the $\bar{k}_{i}$ are nondecreasing, up to affine functions, then there is a constant $C=C(T)$ such that

$$
\left\|u_{1}-u_{2}\right\|_{W^{1.1}([0, T])} \leqslant C\left\|f_{1}-f_{2}\right\|_{W^{1.1}([0, T])} .
$$

(iv) Assume that the $\bar{k}_{i}$ are nondecreasing and $\operatorname{var}\left(a_{i} ; 0, \infty\right)<a_{i}(0)-c$ for some $c>0$, and define $X(f)=\left\{w \in \mathbf{R}\left|\int_{0}^{\infty}\right| \sum_{i} a_{i}(s) \bar{k}_{i}(w)-f^{\prime}(s) \mid d s<\infty\right\}$. If $X(f) \neq$ $\varnothing$, then $u(t) \rightarrow u_{\infty} \in X(f)$ as $t \rightarrow \infty$.

(v) If the $\bar{k}_{i}$ are nondecreasing, $e^{c t} a_{i}(t)$ is nonincreasing for some $c>0$, and $0<\int_{0}^{\infty} a_{i}(s) d s<\infty$, then there are $C_{0}, C_{1}>0$, such that for all $T$

$$
\left\|u_{1}-u_{2}\right\|_{W(T)} \leqslant C_{0}\left\|f_{1}-f_{2}\right\|_{W(T)},
$$

where $\|u\|_{W(T)}=|u(0)|+\int_{0}^{T}\left\{\left|u^{\prime}(t)\right|+C_{1}|u(t)|\right\} d t$.

(vi) Under the assumptions of (v), $\int_{0}^{\infty}\left\{\left|f^{\prime}(t)\right|+\left|f(t)-f_{\infty}\right|\right\} d t<\infty$ implies $\int_{0}^{\infty}\left\{\left|u^{\prime}(t)\right|+\left|u(t)-u_{\infty}\right|\right\} d t<\infty$, where

$$
u_{\infty}+\sum_{i} \int_{0}^{\infty} a_{i}(s) d s \cdot \bar{k}_{i}\left(u_{\infty}\right)=f_{\infty} .
$$

(vii) If the $\bar{k}_{i}$ are nondecreasing, the $a_{i}$ are integrable and nonincreasing, and $\operatorname{meas}\left(\left\{s \leqslant t\left|a_{i}(s)\right| 0\right\}\right) \geqslant t / 2$ for all $t$, then the conclusion of $(\mathrm{v})$ is true.

Also, various comparison results will be shown. In the special case above, assume that the $\bar{k}_{i}$ are nondecreasing and the $a_{i}$ are nonincreasing and nonnegative.

(viii) If $f_{1}(0) \leqslant f_{2}(0)$ and $\left(f_{1}-f_{2}\right)^{\prime} \leqslant 0$ a.e., then $u_{1}(t) \leqslant u_{2}(t)$ for all $t$.

As consequences, asymptotic results can be derived:

(ix) If $a_{i}(\infty)>0, \int_{0}^{\infty}\left(a_{i}(t)-a_{i}(\infty)\right) d t<\infty$, and some of the $\bar{k}_{i}$ are strictly increasing and onto, then limess $\sup _{[t, \infty)}\left|f^{\prime}(\cdot)-\beta\right|=0$ for some $\beta$ implies that $u$ has a limit as $t \rightarrow \infty$.

(x) Assume that $\bar{k}_{i}(0)=0, \bar{k}_{i}(u) \cdot\left[\bar{k}_{i}(u+1)\right]^{-1} \rightarrow 1$ as $|u| \rightarrow \infty, e^{c t} a_{i}(t)$ is nonincreasing for some $c>0$, and $\int_{0}^{\infty}(1+t) \cdot a_{i}(t) d t<\infty$. If $f(\cdot)$ is measurable, bounded, and has a limit at $\infty$, then so does $u(\cdot)$.

\section{REFERENCES}

1. H. Engler, Bounds and asymptotics for a scalar Volterra integral equation, J. Integral Equations 7 (1984), 209-227.

2. G. Gripenberg, An abstract nonlinear Volterra equation, Israel J. Math. 34 (1979), 198-212.

3. __ Bounded solutions of a Volterra equation, J. Differential Equations 28 (1978), 18-22.

4. T. Kiffe and M. Stecher, An abstract Volterra integral equation in a reflexive Banach space, J. Differential Equations 34 (1979), 303-325.

5. __ , $L^{2}$-solutions of Volterra integral equations, SIAM J. Math. Anal. 10 (1979), 274-280.

6. V. Lakshmikantham and S. Leela, Differential and integral inequalities. I, II, Academic Press, New York, 1969.

7. J. J. Levin, A bound on the solutions of a Volterra equation, Arch. Rational Mech. Anal. 52 (1973), 339-349.

8. Some a priori bounds for nonlinear Volterra equations, SIAM J. Math. Anal. 7 (1976), $872-896$.

9. S. O. London, On the solutions of a nonlinear Volterra equation, J. Math. Anal. Appl. 39 (1972), 564-573.

10. R. K. Miller, Nonlinear Volterra integral equations, Benjamin, Menlo Park, 1971.

11. J. T. Schwartz, Nonlinear functional analysis, Gordon \& Breach, New York, 1969. 
12. O. J. Staffans, A bound on the solutions of a nonlinear Volterra equation, J. Math. Anal. Appl. 83 (1981), 127-134.

13. __ A priori bounds for a discontinuous Volterra equation, J. Integral Equations 3 (1981), 231-243.

14. J. H. Roberts and W. R. Mann, On a certain nonlinear integral equation of the Volterra type, Pacific J. Math. 1 (1951), 431-445.

15. J. J. Levin, Remarks on a Volterra equation, Delay and Functional Differential Equations and their Applications, Academic Press, London, New York, 1972, pp. 233-255.

16. O. J. Staffans, $A$ note on a Volterra equation with several nonlinearities, J. Integral Equations 7 (1984), 249-252.

17. H. Engler, A note on scalar Volterra integral equations. II, J. Math. Anal. Appl. (to appear).

Department of Mathematics, Georgetown University, Washington, D.C. 20057 